\title{
Evaluation of internal derangement of the temporomandibular joint by panoramic radiographs compared with magnetic resonance imaging
}

\author{
Sug-Joon Ahn, ${ }^{\text {a }}$ Tae-Woo Kim, ${ }^{b}$ Dong-Yul Lee, ${ }^{c}$ and Dong-Seok Nahm ${ }^{d}$ \\ Seoul, South Korea
}

Introduction: Panoramic radiographs are routinely used in orthodontic diagnosis and treatment planning. It would be useful if these radiographs could also provide information about internal derangement (ID) of the temporomandibular joint (TMJ). Methods: The sample consisted of 168 condyles in 84 women. They underwent panoramic radiography on 1 of 3 machines and bilateral magnetic resonance imaging of the TMJ on 1 machine. Six panoramic variables were analyzed by 2-factor ANOVA to evaluate differences in condylar morphology with respect to status of the TMJ ID and type of panoramic machine. Results: Decreased condylar heights and distally inclined condyles were found in the patients with TMJ ID, irrespective of the type of panoramic machine used. The change became more severe as ID progressed to disk displacement without reduction. Conclusions: This study suggests that some panoramic variables can help the clinician identify patients with potential ID. (Am J Orthod Dentofacial Orthop 2006;129:479-85)

$\mathbf{P}$ anoramic radiography is routinely used in orthodontics because it provides general information about teeth, mandible, and other regions of the jaw. Panoramic radiographs also yield replicable results, have a favorable cost-benefit relationship, and expose patients to relatively low doses of radiation. ${ }^{1-3}$

Various imaging modalities are available for assessing changes in temporomandibular joint (TMJ) morphology and function, ${ }^{4}$ including plain radiography, tomography, computed tomography, arthrography, and magnetic resonance imaging (MRI). Conventional radiographic methods are generally recommended initially, with the more sophisticated methods reserved for certain patients. Panoramic radiography has been used as the initial imaging technique for TMJ screening

aAssistant Professor, Department of Orthodontics, Dental Research Institute and College of Dentistry, Seoul National University, Seoul, South Korea.

bAssociate professor and chairman, Department of Orthodontics, Dental Research Institute and College of Dentistry, Seoul National University, Seoul, South Korea.

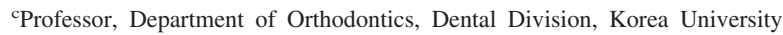
Guro Hospital, Seoul, South Korea.

${ }^{\mathrm{d}}$ Professor, Department of Orthodontics, Dental Research Institute and College of Dentistry, Seoul National University, Seoul, South Korea.

Supported by a grant of the Korea Health 21 R\&D Project, Ministry of Health \& Welfare, Republic of Korea (03-PJ1-PG1-CH09-0001).

Reprint requests to: Tae-Woo Kim, Associate Professor and Chairman, Department of Orthodontics, College of Dentistry, Seoul National University, 28-22 Yunkeun-Dong, Chongro-Ku, Seoul 110-768, Korea (ROK); e-mail, taewoo@ snu.ac.kr.

Submitted, July 2004; revised and accepted, September 2004. 0889-5406/\$32.00

Copyright (C) 2006 by the American Association of Orthodontists. doi:10.1016/j.ajodo.2005.12.009 when the clinical examination suggests some form of joint pathology.

However, the use of panoramic radiographs for TMJ imaging is still controversial. ${ }^{5}$ Some studies found the method reliable in depicting condyles, ${ }^{6,7}$ whereas others showed that the findings on panoramic radiographs were not related to clinical TMJ signs and symptoms, possibly because of misinterpretation of distortion effects as signs of temporomandibular disorders. ${ }^{8,9}$ Panoramic radiography is not usually used in diagnosing internal derangement (ID) of the TMJ because of its inability to visualize disk, joint space, and fossa.

Recently, bony changes were found on the articular surface of the mandibular condyle in patients with TMJ ID. ${ }^{10,11}$ In addition, deviations in form and morphology are more common in the lateral third of the condyle. ${ }^{12}$ This means that panoramic radiography might be used to diagnose TMJ ID, because it depicts the lateral and medial thirds of the condylar head. ${ }^{13}$ If some characteristic findings from panoramic radiography suggest ID, this might further increase the diagnostic value of panoramic radiography in orthodontics.

Quantitative measurements on panoramic radiography are difficult because of magnification differences and image distortions. ${ }^{14}$ However, the reproducibility of vertical and angular measurements is relatively acceptable if the patient's head is correctly positioned in the equipment. ${ }^{15,16}$ In this regard, some quantitative methods have been used for linear measurements, such 
as tooth length, ${ }^{15}$ edentulous ridge height, ${ }^{14}$ and mandibular and condylar dimensions. ${ }^{5,17}$ We measured the panoramic variables quantitatively, and the results were compared with those of an MRI. The aim of this study was to find the panoramic variables that provide information on TMJ ID independent of the panoramic machine, by using MRI as the gold standard.

\section{MATERIAL AND METHODS}

Our subjects were women over the age of 17. Men were not included to prevent skewing the measurements with sex-related size differences. None of the subjects had previously been diagnosed with juvenile rheumatoid arthritis. Each subject had a primary complaint of malocclusion, and a routine panoramic radiograph was taken for the orthodontic records. Irrespective of TMJ status, each subject consented to a bilateral high-resolution MRI in the sagittal (opened and closed) and coronal (closed) planes to evaluate the TMJ. The institutional review board of the University Hospital approved the research protocol.

The MRIs were obtained with a Signa Horizon (GE, Waukesha, Wis) operating at $1.5 \mathrm{~T}$ and a unilateral 3-in surface receiver coil (GE). Initially, the axial scout images were obtained at the level of the TMJ to identify the long axes of the condyles. The nonorthogonal sagittal sections were obtained perpendicular to the condyles, and the nonorthogonal coronal oblique sections were also obtained. The closed-mouth images were obtained at maximum dental intercuspation, and the open-mouth images were taken at maximum unassisted vertical mandibular opening by using a Burnett bidirectional TMJ device (Medrad, Pittsburgh, Pa). T1-weighted 600/12 (repetition time [TR] ms/echo time [TE] ms) and proton-density 4000/14 (TR ms/TE $\mathrm{ms}$ ) pulse sequences were performed in the sagittal plane by using a $3-\mathrm{mm}$ slice thickness, a $10-\mathrm{cm}$ field of view, 2 excitations, and an image matrix of $254 \times 192$ pixels. T1-weighted 500/12 (TR ms/TE ms) pulse sequence was performed in the coronal plane under the same conditions.

A radiologist experienced in interpreting TMJ MRIs and an orthodontist interpreted the images. The TMJ disc position was divided into 3 categories according to the following criteria.

1. Normal disc position (Fig $1, A$ and $B$ ). In the closed-mouth position, the intermediate zone of the disc was interposed between the condyle and the posterior slope of the articular eminence, with anterior and posterior bands equally spaced on either side of the condylar load point.
2. Disc displacement with reduction (DDR). The disc was anteriorly displaced relative to the posterior slope of the articular eminence and the head of the condyle (Fig 1,C). However, the disc was reduced on mouth opening (Fig 1,D).

3. Disc displacement without reduction (DDNR). The disc was anteriorly displaced relative to the posterior slope of the articular eminence and the head of the condyle (Fig 1,E), but without reduction of the disc on mouth opening (Fig 1,F).

The position of the disc was evaluated carefully, and some images without the clarity necessary for diagnosis of TMJ ID were rejected. A total of 119 patients were originally selected and analyzed.

The panoramic radiographs were obtained with 1 of 3 machines: Auto 2000 (Asahi Roentgen, Kyoto, Japan), Cranex DC 3 (Soredex, Orion, Helsinki, Finland), or Orthophos Plus (Sinora, Siemens, Bensheim, Germany). The patient's head was exposed in an optimum position according to the operating instructions. Only the films on which the mandibular condyles could be completely visualized were used in the study. Approximately one third of the original films (35 patients) were rejected and the final sample consisted of 168 joints of the right and left sides in 84 women (mean age, 23.5 years; range, 17.4-43.6 years).

The outlines of the condyle and the ramus on both sides of the panoramic radiograph were traced on acetate paper. A single investigator (S-J.A.) traced all radiographs. The tracings were digitized with a digitizer interfaced with a desktop computer. Seven landmarks were digitized on each side, from which 6 variables (3 linear, 1 angular, and 2 ratio measurements) were calculated from each side. The linear measurements consisted of ramus height, condylar height, and condylar head height, the angular measurement was condylar head angle, and the ratio measurements were condylar-head to ramus-height ratio and condylar-head-height to ramus-height ratio (Fig 2, Table). The positions of all landmarks and their measurements are shown in Figure 2. To analyze differences in the variables and the interaction effects with respect to status of the TMJ ID and type of panoramic machine, 2-factor ANOVA was used. The Duncan multiple comparisons test was performed at a significance level of $\alpha=0.05$ (Table).

To test the magnitude of the measurement error, the panoramic radiographs of 15 randomly selected patients were measured again. According to Dahlberg's formula, ${ }^{18}$ the errors were 0.21 to $0.95 \mathrm{~mm}$ for the linear measurements, $0.92^{\circ}$ for the angular mea- 

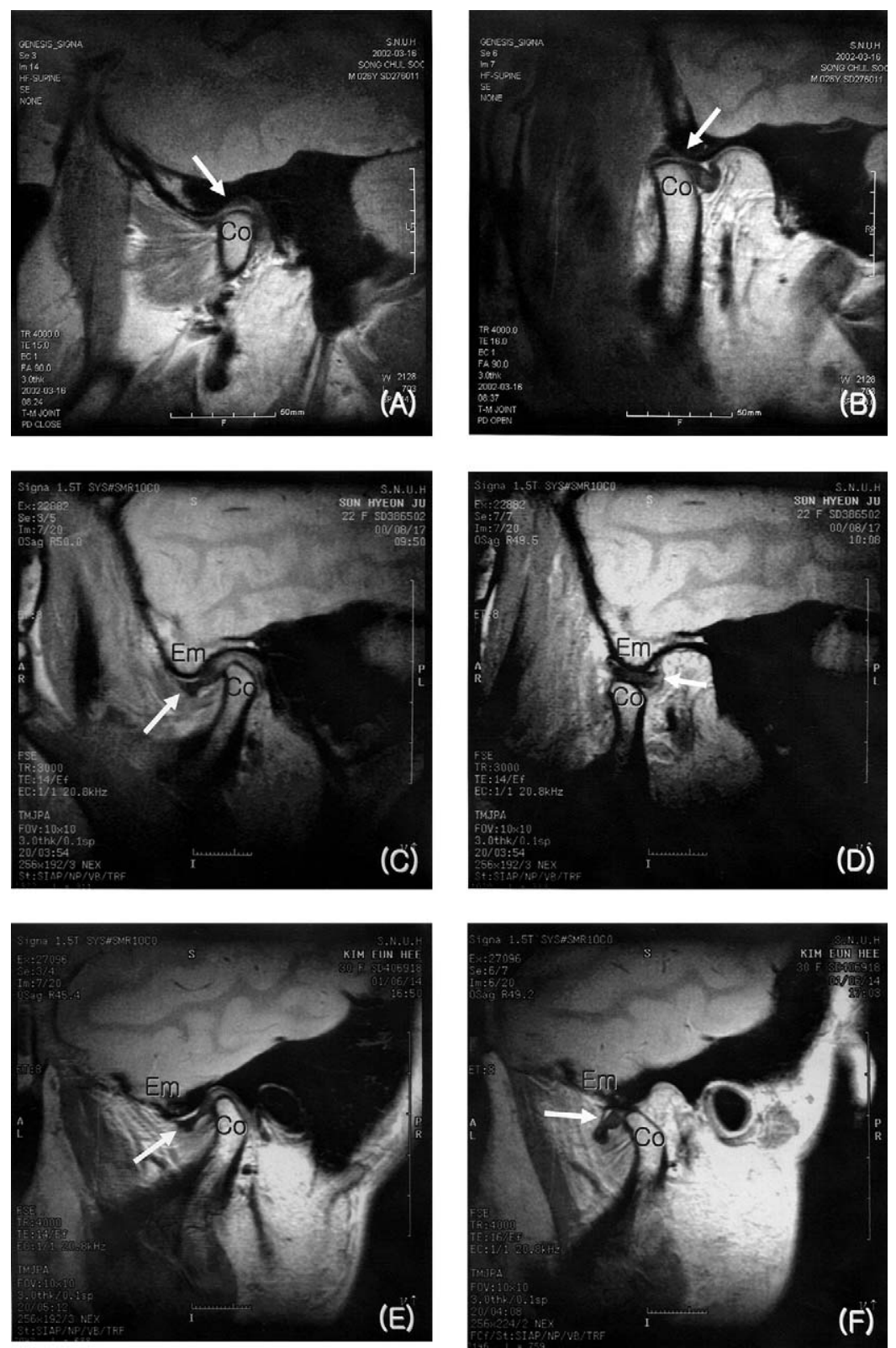

Fig 1. Normal TMJ disc position to condyle in $\mathbf{A}$, closed-mouth, and $\mathbf{B}$, open-mouth sagittal images. Intermediate zone of disc (arrow) was interposed between condyle (Co) in both positions. DDR to condyle in $\mathbf{C}$, closed-mouth, and $\mathbf{D}$, open-mouth sagittal images. Disc (arrow) was anteriorly displaced relative to posterior slope of articular eminence $(E m)$ and head of condyle (Co). However, disc was reduced on mouth opening. DDNR to condyle in $\mathbf{E}$, closed-mouth, and $\mathbf{F}$, open-mouth sagittal images. Disc (arrow) was anteriorly displaced relative to posterior slope of articular eminence $(\mathrm{Em})$ and head of condyle $(\mathrm{Co})$, but without reduction of disc on mouth opening.

surements, and 0.35 to 0.80 for the ratio measurements.

\section{RESULTS}

The Table shows the differences in the panoramic variables with respect to the status of TMJ ID and type of panoramic machine. The left and right panoramic variables were pooled, because there was no marked statistical difference between left and right condyles. The 2-factor ANOVA showed that the status of TMJ ID had significant effects on the panoramic variables except for ramus height, 


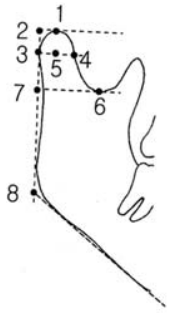

(A)

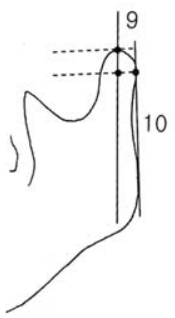

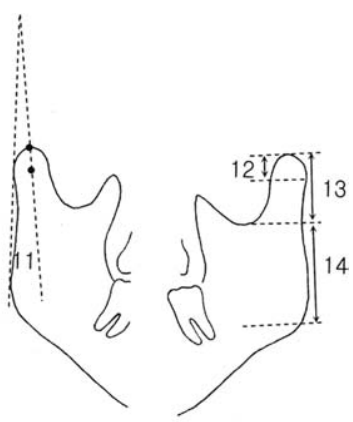

(B)
Fig 2. A, Landmarks and reference planes, and B, measurements used in this study: 1 , most superior point of condylar head; 2, point intersecting perpendicular projection of point 1 and ramus tangent; 3, outermost point of condylar head; 4 , point intersecting perpendicular projection of point 3 and inner condylar outline; 5 , midpoint between points 3 and 4; 6, deepest point between coronoid process and condylar process; 7 , perpendicular projection of point 6 on ramus tangent; 8 , intersection between ramus tangent and inferior mandibular line; 9, line connecting points 1 and 5 (condylar axis); 10, ramus tangent; 11, angle between condylar axis and ramus tangent (condylar head angle); 12, distance between points 2 and 3 (condylar head height); 13, distance between points 2 and 7 (condylar height); 14, distance between points 7 and 8 (ramus height); 15, ratio between condylar head height and ramus height; and 16 , ratio between condylar height and ramus height.

whereas type of panoramic machine had no significant effect on the panoramic variables except for condylar height. This indicates that some variables can provide information on TMJ ID, irrespective of the type of panoramic machine.

The statistical differences among the status of TMJ ID were mainly due to discrepancies between normal disc position and DDNR. Condylar head height, condylar head angle, and condylar-head-height to ramus-height ratio showed that skeletal changes evidently appear when ID progresses to DDNR (Table). The subjects with DDNR had small and distally inclined condyles compared with those with either normal disc position or DDR. Only condylar height and condylar-height to ramus-height ratio identified the differences among normal disc position, DDR, and DDNR, although there were some differences according to type of panoramic machine.

The panoramic values in the Orthophos Plus machine were generally small compared with the other panoramic machines (Table). This could be explained by differences in magnification, because different pan- oramic machines have different projection geometry. However, the relationships between TMJ ID and panoramic variables were not influenced; this might be due to the uniform relationship between TMJ ID and panoramic variables irrespective of the panoramic machine (Table). It could also be due to the relatively small difference in panoramic values among the machines.

The interaction effect between type of panoramic machine and status of ID was shown only in ramus height. This means that ramus height can be influenced by interaction between the type of panoramic machine and the status of ID. This can be explained by the fact that ramus height is influenced by various factors, such as the depth and position of the mandibular notch, and the inclination of the ramus and the ramus tangent. However, ramus height did not show statistical significance with respect to the type of panoramic machine and the status of TMJ ID.

\section{DISCUSSION}

Dental panoramic radiography is a good technique to visualize the bony components of the TMJ and the ascending rami in the mandible because of cost and clinical effectiveness. ${ }^{1,2,5}$ Although panoramic radiography depicts only the lateral and medial thirds of the condylar head, ${ }^{13}$ bony lesions occur more frequent in the lateral third. ${ }^{12}$ In addition, panoramic radiography has been reported to be reliable in depicting the condyle and in screening for TMJ abnormalities. 6 ,7

However, image distortion and projection artifacts are limitations as a result of the nonlinear variation in the magnification at the different object depths. ${ }^{15,16,19}$ These distortion and projection artifacts can be misinterpreted as signs of TMD. ${ }^{8,9}$ Even though the use of panoramic radiography is connected to those methodological pitfalls, some quantitative methods have been used to measure the vertical dimension, the angle, and the ratio. ${ }^{5,15-17}$ These dimensions are known to be relatively reliable compared with the horizontal dimension. In this study, 3 vertical, 1 angular, and 2 ratio measurements on panoramic radiography were used to evaluate the association between TMJ ID and the panoramic findings.

We also found some differences in the linear measurements according to type of panoramic machine (Table). The linear measurements of the Orthophos Plus machine were smaller than those of the other 2 machines. However, these differences did not influence the relationships between the status of TMJ ID and the panoramic variables, because the relationship was uniform irrespective of the machine.

This study showed that TMJ ID is associated with 
Table. Panoramic variables with respect to type of panoramic machine and status of TMJ ID

\begin{tabular}{|c|c|c|c|c|c|c|}
\hline $\begin{array}{l}\text { Panoramic } \\
\text { variables }\end{array}$ & Panoramic & Normal $(n=68)$ & $\operatorname{DDR}(n=56)$ & $\operatorname{DDNR}(n=44)$ & Total $(n=168)$ & Significance $^{a}$ \\
\hline \multirow{4}{*}{$\begin{array}{r}\text { Condylar head } \\
\text { height }(\mathrm{mm})\end{array}$} & Or $(\mathrm{n}=34)$ & $8.3 \pm 3.6$ & $7.8 \pm 1.8$ & $4.1 \pm 2.4$ & $7.1 \pm 3.3$ & \multirow{4}{*}{$\begin{array}{c}\text { Normal }=\mathrm{DDR} \\
>\mathrm{DDNR}^{* * *} \\
\mathrm{Or}=\mathrm{Au}=\mathrm{Cr}^{\mathrm{NS}}\end{array}$} \\
\hline & $\mathrm{Au}(\mathrm{n}=68)$ & $9.8 \pm 2.6$ & $9.2 \pm 2.4$ & $4.5 \pm 2.6$ & $7.9 \pm 3.4$ & \\
\hline & $\mathrm{Cr}(\mathrm{n}=68)$ & $8.4 \pm 2.4$ & $7.0 \pm 2.9$ & $5.3 \pm 2.2$ & $7.5 \pm 3.2$ & \\
\hline & Total $(\mathrm{n}=168)$ & $8.9 \pm 2.8$ & $8.2 \pm 2.7$ & $4.7 \pm 2.4$ & $7.5 \pm 3.2$ & \\
\hline \multirow{4}{*}{$\begin{array}{l}\text { Condylar height } \\
\text { (mm) }\end{array}$} & Or $(\mathrm{n}=34)$ & $24.5 \pm 6.3$ & $20.3 \pm 2.6$ & $17.2 \pm 2.7$ & $20.9 \pm 5.2$ & \multirow{4}{*}{$\begin{array}{c}\text { Normal }>\mathrm{DDR} \\
>\mathrm{DDNR} * * \\
\mathrm{Or}<\mathrm{Au}=\mathrm{Cr}^{*}\end{array}$} \\
\hline & $\mathrm{Au}(\mathrm{n}=68)$ & $25.7 \pm 4.1$ & $23.8 \pm 3.6$ & $18.7 \pm 4.5$ & $22.9 \pm 4.9$ & \\
\hline & $\mathrm{Cr}(\mathrm{n}=68)$ & $25.6 \pm 5.4$ & $23.2 \pm 4.6$ & $19.7 \pm 3.7$ & $23.7 \pm 5.1$ & \\
\hline & Total $(\mathrm{n}=168)$ & $25.2 \pm 5.2$ & $23.2 \pm 4.0$ & $18.8 \pm 3.6$ & $22.8 \pm 5.1$ & \\
\hline \multirow[t]{4}{*}{ Ramus height (mm) } & Or $(\mathrm{n}=34)$ & $45.3 \pm 4.7$ & $48.4 \pm 6.1$ & $42.3 \pm 4.9$ & $45.2 \pm 5.6$ & \multirow{4}{*}{$\begin{array}{l}\text { Normal }=\mathrm{DDR} \\
\quad=\mathrm{DDNR}^{\mathrm{NS}} \\
\mathrm{Or}=\mathrm{Au}=\mathrm{Cr}^{\mathrm{NS}}\end{array}$} \\
\hline & $\mathrm{Au}(\mathrm{n}=68)$ & $45.1 \pm 5.8$ & $45.6 \pm 4.6$ & $42.7 \pm 5.1$ & $44.4 \pm 5.2$ & \\
\hline & $\mathrm{Cr}(\mathrm{n}=68)$ & $45.1 \pm 5.5$ & $43.6 \pm 4.6$ & $47.1 \pm 4.8$ & $45.1 \pm 5.1$ & \\
\hline & Total $(\mathrm{n}=168)$ & $44.9 \pm 5.4$ & $45.4 \pm 5.1$ & $43.8 \pm 5.7$ & $44.8 \pm 5.4$ & \\
\hline \multirow{4}{*}{$\begin{array}{l}\text { Condylar head } \\
\text { angle }\left(^{\circ}\right)\end{array}$} & Or $(\mathrm{n}=34)$ & $-0.2 \pm 10.7$ & $4.7 \pm 10.0$ & $23.5 \pm 10.2$ & $7.0 \pm 13.9$ & \multirow{4}{*}{$\begin{array}{c}\text { Normal }=\mathrm{DDR} \\
<\mathrm{DDNR} * * * \\
\mathrm{Or}=\mathrm{Au}=\mathrm{Cr}^{\mathrm{NS}}\end{array}$} \\
\hline & $\mathrm{Au}(\mathrm{n}=68)$ & $-0.3 \pm 7.6$ & $0.5 \pm 8.3$ & $18.0 \pm 10.9$ & $6.1 \pm 10.3$ & \\
\hline & $\mathrm{Cr}(\mathrm{n}=68)$ & $1.8 \pm 9.0$ & $4.4 \pm 11.0$ & $11.2 \pm 9.3$ & $4.7 \pm 10.4$ & \\
\hline & Total $(\mathrm{n}=168)$ & $0.8 \pm 9.0$ & $2.5 \pm 9.8$ & $16.7 \pm 2.6$ & $5.7 \pm 12.7$ & \\
\hline \multirow{4}{*}{$\begin{array}{c}\text { Condylar head } \\
\text { height/ramus } \\
\text { height }(\%)\end{array}$} & Or $(\mathrm{n}=34)$ & $18.7 \pm 8.0$ & $16.0 \pm 2.7$ & $9.4 \pm 5.3$ & $15.7 \pm 7.0$ & \multirow{4}{*}{$\begin{array}{c}\text { Normal }=\mathrm{DDR} \\
>\mathrm{DDNR} * * * \\
\mathrm{Or}=\mathrm{Au}=\mathrm{Cr}^{\mathrm{NS}}\end{array}$} \\
\hline & $\mathrm{Au}(\mathrm{n}=68)$ & $22.1 \pm 6.7$ & $20.2 \pm 5.0$ & $10.5 \pm 5.4$ & $17.8 \pm 7.5$ & \\
\hline & $\mathrm{Cr}(\mathrm{n}=68)$ & $18.9 \pm 6.1$ & $16.1 \pm 5.8$ & $11.2 \pm 4.2$ & $16.3 \pm 6.3$ & \\
\hline & Total $(\mathrm{n}=168)$ & $19.9 \pm 6.8$ & $17.9 \pm 5.3$ & $10.5 \pm 4.9$ & $16.8 \pm 7.1$ & \\
\hline \multirow{4}{*}{$\begin{array}{l}\text { Condylar height/ } \\
\text { ramus height }(\%)\end{array}$} & Or $(\mathrm{n}=34)$ & $52.7 \pm 13.3$ & $42.7 \pm 8.3$ & $41.2 \pm 8.2$ & $46.8 \pm 11.8$ & \multirow{4}{*}{$\begin{array}{c}\text { Normal }>\text { DDR } \\
>\mathrm{DDNR}^{* * *} \\
\mathrm{Or}=\mathrm{Au}=\mathrm{Cr}^{\mathrm{NS}}\end{array}$} \\
\hline & $\mathrm{Au}(\mathrm{n}=68)$ & $58.5 \pm 16.3$ & $52.7 \pm 9.1$ & $46.0 \pm 15.0$ & $52.6 \pm 14.7$ & \\
\hline & $\mathrm{Cr}(\mathrm{n}=68)$ & $58.5 \pm 19.4$ & $54.9 \pm 11.1$ & $42.5 \pm 8.3$ & $53.8 \pm 16.5$ & \\
\hline & Total $(\mathrm{n}=168)$ & $57.3 \pm 17.2$ & $51.5 \pm 10.5$ & $43.9 \pm 12.5$ & $51.9 \pm 15.0$ & \\
\hline
\end{tabular}

$D D R$, Disc displacement with reduction; $D D N R$, disc displacement without reduction; Or, Orthphos plus, Sinora, Siemens, Bensheim, Germany; $\mathrm{Au}$, Auto 2000, Ashahi Roentgen, Kyoto, Japan; $\mathrm{Cr}$, Cranex DC 3, Soredex, Orion Corporation, Helsinki, Finland.

${ }^{a}$ Two-way factorial ANOVA and Duncan's multiple comparisons were used to evaluate differences in condylar morphology with respect to status of TMJ ID and type of panoramic machine: NS, not significant; $* \mathrm{P}<.05$; ** $\mathrm{P}<.01$; *** $\mathrm{P}<.001$. Duncan's method at significance level of $\alpha$ $=0.05$ used for multiple comparisons between different groups.

skeletal changes in the mandibular condyle on panoramic radiography, although different panoramic machines were used. The subjects with DDNR had statistically significantly lower condylar heights and distally inclined condyles compared with those with DDR or normal disc position, irrespective of the panoramic machine. The subjects with DDR also had slightly less condylar height and greater condylar head angles than those with normal disc positions, but the amount of change was less than that between DDR and DDNR (Table). Only condylar height and condylar-height to ramus-height ratio were statistically significant between normal disk position and DDR. This is consistent with previous studies. ${ }^{10,11,20}$ Morphological changes of the mandibular condyle are reported in patients with TMJ ID. Kurita et $\mathrm{al}^{10,11}$ evaluated resorption of the superolateral pole of the condyle using MRI. They reported that resorption of the superolateral pole of the condyle was highly prevalent in TMJs with advanced disc displacement and disc deformation.

It appears that the condyle begins to change while ID develops to DDR from normal disc position, because condylar height begins to change from normal disc position to DDR. This was verified by a decrease in the condylar-height to ramus-height ratio in the subjects with DDR compared with those with normal disc positions. More substantial changes can occur in the condylar region, if ID progresses to DDNR. The subjects with DDNR showed changes in condylar shape and loss of the vertical dimension as a result of severe erosion and hard-tissue remodeling. Small and distally inclined condyles were evident in the subjects with DDNR (Fig 3). This means that the effects of DDR were of lesser magnitude, without severe deformation compared with those with DDNR. Hard-tissue remodeling and bony degenerative changes were reported to be the radiographic characteristics of the final stage in TMJ ID. ${ }^{21}$

This study showed an association between TMJ ID and panoramic variables, although the cause-andeffect relationship between TMJ ID and changes in panoramic variables is unclear. Altered preexisting condylar shape might cause TMJ ID, or TMJ ID might cause altered condylar shape. Some patients might have TMJ ID even before condylar degeneration. Nevertheless, this study suggests that TMJ ID 


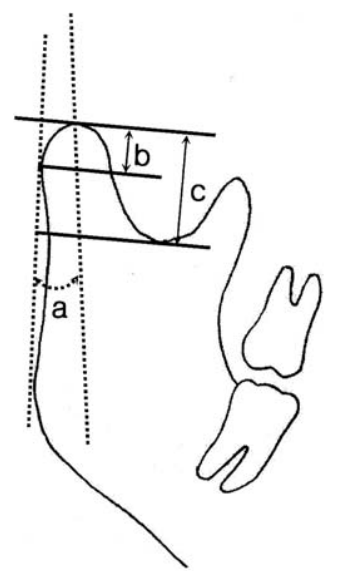

(A)

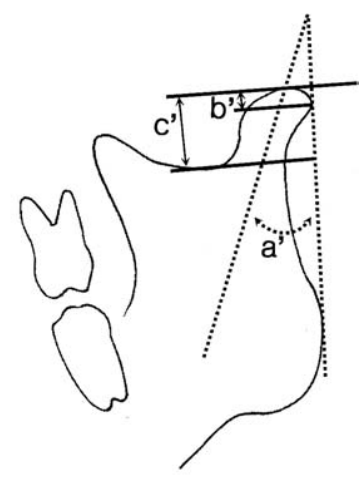

(B)
Fig 3. Examples of joints. A, Normal joint; B, joint with DDNR. External resorption, severe erosion, and hardtissue remodeling can occur when ID progresses to DDNR. This might induce increase in condylar angle $\left(a^{\prime}\right)$ and decreases in condylar head height $\left(b^{\prime}\right)$ and condylar height $\left(c^{\prime}\right)$, compared to patients with condyles having normal disc positions ( $a, b$, and $c$ ).

can affect mandibular morphology_particularly the mandibular condyle. The subjects with DDR showed decreased condylar heights compared with those with normal disc positions. These changes became increasingly worse as the subjects' conditions progressed to DDNR. This was supported by previous studies; patients with DDNR had osseous changes in the condylar heads, but these changes were less frequent in patients with DDR. ${ }^{22,23}$

Although it has some shortcomings, such as the inability to visualize the soft structures, and distortion and magnification of the image, ${ }^{1,3}$ panoramic radiography is recommended as the initial imaging technique for assessing the TMJ when a clinical examination suggests some form of joint pathology. In addition, panoramic radiograph should be taken routinely before orthodontic treatment. These were the main reasons for choosing panoramic radiography in this study. We suggest that a quantitative evaluation of the panoramic radiography can provide valuable information to supplement that obtained from a qualitative evaluation for an initial assessment of TMJ ID, despite the shortcomings. Women with decreased condylar heights and distally inclined condyles must be screened carefully, because they might have TMJ ID. In addition, the type of panoramic machine did not influence these results. It is possible to diagnose TMJ ID accurately based on the combined evidence from the panoramic radiographs and the patient's history and physical examination.

\section{CONCLUSIONS}

This study was performed to analyze the relationship between TMJ ID and panoramic variables by using MRI as the gold standard. We found some differences in condylar morphology and shape in the subjects with TMJ ID compared with those with normal TMJs. Decreased condylar height and distally inclined condyles were found in the patients with ID. These changes became increasingly severe as ID progressed to DDNR via DDR. In addition, these changes were found irrespective of the type of panoramic machine used in this study. Our results suggest that TMJ ID might play a role in the changes in the condyle, and some panoramic variables can assist clinicians in discriminating between patients with potential ID.

\section{REFERENCES}

1. Habets LLMH, Bezuur JN, Jimenez Lopez V, Hansson TL. The OPG: an aid in TMJ diagnostics. III. A comparison between lateral tomography and dental rotational panoramic radiography (orthopantomography). J Oral Rehabil 1989;16: 401-6.

2. Habets LLMH, Bezuur JN, Naeije M, Hansson TL. The orthopantomogram, an aid in diagnosis of temporomandibular joint problems. II. The vertical symmetry. J Oral Rehabil 1988;15: 465-71.

3. Mongini $F$. The importance of radiography in the diagnosis of TMJ dysfunctions. A comparative evaluation of transcranial radiographs and serial tomography. J Prosthet Dent 1981;45:186-98.

4. Westesson PL. Reliability and validity of imaging diagnosis of temporomandibular joint disorder. Adv Dent Res 1993;7:137-51.

5. Habets LLMH, Bezuur JN, van Ooij CP, Hansson TL. The orthopantomogram, an aid in diagnosis of temporomandibular joint problems. I. The factor of vertical magnification. J Oral Rehabil 1987;14:475-80

6. Larheim TA, Johannessen S, Tveito L. Abnormalities of temporomandibular joint in adults with rheumatic disease. A comparison of panoramic, transcranial and transpharyngeal radiography with tomography. Dentomaxillofac Radiol 1988;17: 109-13.

7. Kononen M. Subjective symptoms from the stomatognathic system in patients with psoriatic arthritis. Acta Odontol Scand 1986;44:377-83.

8. Bush FM, Harrington WG, Harkins SW. Interexaminer comparision of bone scintigraphy and panoramic radiography of temporomandibular joints: correlation with signs and symptoms. J Prosthet Dent 1992;67:246-51.

9. Ruf S, Pancherz H. Is orthopantomography reliable for TMJ diagnosis? An experimental study on a dry skull. J Orofac Pain 1995;9:365-74.

10. Kurita H, Ohtsuka A, Kobayashi H, Kurashina K. Resorption of the lateral pole of the mandibular condyle in temporomandibular disc displacement. Dentomaxillofac Radiol 2001;30: 88-91. 
11. Kurita H, Ohtsuka A, Kobayashi H, Kurashina K. Relationship between increased horizontal condylar angle and resorption of the posterosuperior region of the lateral pole of the mandibular condyle in temporomandibular joint internal derangement. Dentomaxillofac Radiol 2003;32:26-9.

12. Oberg T, Carlsson GE, Fajers CM. The temporomandibular joint. A morphologic study on a human autopsy material. Acta Odontol Scand 1971;29:349-84.

13. Hollender L. Imaging of the TMJ: the value of conventional radiography and standard views and tomograms. In: Worthington R, Evans JR, editors. Controversies in oral and maxillofacial surgery. Philadelphia: W. B. Saunders; 1994. p. 25-40.

14. Ramstad T, Hensten-Pettersen O, Mohn E, Ibrahim SI. A methodological study of errors in vertical measurements of edentulous ridge height on orthopantomographic radiograms. J Oral Rehabil 1978;5:403-12.

15. Larheim TA, Svanaes DB, Johannessen S. Reproducibility of radiographs with the orthopantomograph 5: tooth length assessment. Oral Surg Oral Med Oral Pathol 1984;58:736-41.

16. Larheim TA, Svanaes DB. Reproducibility of rotational panoramic radiography: mandibular linear dimensions and angles. Am J Orthod Dentofacial Orthop 1986;90:45-51.
17. Kjellberg H, Ekestubbe A, Kiliaridis S, Thilander B. Condylar height on panoramic radiographs. A methodological study with a clinical application. Acta Odontol Scand 1994;52:43-50.

18. Dahlberg G. Statistical methods for medical and biological students. New York: Interscience Publications; 1940.

19. Lund TM, Manson-Hing LR. A study of focal troughs of three panoramic dental X-ray machines. Part I. the area of sharpness. Oral Surg Oral Med Oral Pathol 1975;39:318-28.

20. Westesson PL, Bifano JA, Tallents RH, Hatala MP. Increased horizontal angle of the mandibular condyle in abnormal temporomandibular joints. A magnetic resonance imaging study. Oral Surg Oral Med Oral Pathol 1991;7:359-63.

21. Rasmussen OC. Description of population and progress of symptoms in a longitudinal study of temporomandibular joint arthropathy. Scand J Dent Res 1981;89:196-203.

22. Anderson QN, Katzberg RW. Pathologic evaluation of disc dysfunction and osseous abnormalities of the temporomandibular joint. J Oral Maxillofac Surg 1985;43:947-51.

23. Westesson PL. Structural hard-tissue changes in temporomandibular joint with internal derangement. Oral Surg Oral Med Oral Pathol 1985;59:220-4. 